\title{
Resolução de Problemas de Divisão Inexata a partir de Reflexões sobre o Significado do Resto
}

\author{
Sintria Labres Lautert ${ }^{1}$ \\ Alina Galvão Spinillo \\ Departamento de Psicologia e da Pós-Graduação em Psicologia Cognitiva da Universidade \\ Federal de Pernambuco, Recife, PE, Brasil \\ Coordenação do Núcleo de Pesquisa em Psicologia da Educação Matemática, \\ Recife, PE, Brasil
}

\begin{abstract}
Resumo
Considerando que os invariantes lógicos da divisão envolvem as relações entre os seus termos e que o resto é um dos termos da divisão, é possível supor que uma forma de desenvolver a compreensão acerca deste conceito seja refletir acerca do significado do resto na resolução de problemas de divisão inexata. A presente investigação examinou esta possibilidade em um estudo de intervenção com crianças que apresentavam dificuldades com o conceito de divisão. Cem crianças (entre 8 e 11 anos), alunas do $4^{\circ}$ ano do Ensino Fundamental de escolas públicas, após um pré-teste, foram alocadas em dois grupos equivalentes quanto às dificuldades que apresentavam com a divisão. Ao grupo experimental (GE) foi oferecida uma intervenção baseada em problemas de divisão inexata que envolvia discussões sobre as formas apropriadas e inapropriadas de lidar com o resto, e as relações deste elemento com os demais termos. Um pós-teste foi aplicado aos participantes, cujos resultados mostraram que a intervenção auxiliou os alunos do GE a superar as dificuldades iniciais identificadas. Ao manipular o resto, a criança é levada a estabelecer comparações entre o tamanho do resto e o número/tamanho das partes, tendo como âncora o princípio da igualdade entre as partes, princípio fundamental da divisão. Neste sentido, entende-se que o resto pode ser considerado uma ferramenta didática capaz de, quando colocado em evidência, auxiliar a criança a desenvolver noções mais elaboradas sobre o conceito de divisão.
\end{abstract}

Palavras-chave: Crianças, divisão inexata, significado do resto, intervenção.

\section{Solving Word Division Problems by Reflecting on the Role Played by the Remainder}

\begin{abstract}
Children's difficulties with division are well known in the literature. One may ask whether these difficulties would be overcome if children had concentrated experience with problem-solving situations involving discussions about the role played by the remainder. This idea was tested in an intervention study carried out with 100 third grade low-income Brazilian children (8 to 11 years old) who experienced difficulties with division. They were equally assigned to an experimental and a control group. All
\end{abstract}

Endereço para correspondência: Universidade Federal de Pernambuco, Centro de Filosofia e Ciências Humanas, Programa de Pós-Graduação em Psicologia Cognitiva, Av. Arquitetura, s/n, $8^{\circ}$ andar, Cidade Universitária, Recife, PE, Brasil 50740-550. Fone: (81) 2126-8272; Fax: (81) 2126-7330. E-mail: sintrialautert@gmail.com As autoras agradecem o apoio recebido do Conselho Nacional de Desenvolvimento Científico e Tecnológico $(\mathrm{CNPq})$ sob forma de bolsa de estudos para realização de doutorado da primeira autora sob orientação da segunda. 
participants were given a pre- and a post-test consisting of 12 division word problems. Children in the experimental group were given an intervention involving a large variety of problem solving situations. They were asked to discuss their thought processes while the examiner explicitly talked with them about the remainder and the equality of the parts. No significant differences were found among the groups in the pre-test. After the intervention, children in the experimental group gave more correct responses than in the pre-test and were also able to offer justifications expressing an understanding of the operational invariants in the concept of division. The control group showed no improvement when compared in the two testing occasions. The conclusion was that discussion explicitly focusing on the role played by the remainder and on the inverse relations can help children overcome their difficulties with the concept of division.

Keywords: Children, division word problems, remainder, intervention.

\section{Resolución de Problemas de División Inexacta através de la Reflexión del Significado del Resto}

\section{Resumen}

Teniendo en cuenta que los invariantes lógicos de la división involucran las relaciones que existen entre sus términos, es posible pensar que una manera de promover la comprensión de eso concepto es llamando la atención del niño sobre el significado del resto en la resolución de problemas de la división inexacta. Así, se ha objetivado investigar esa posibilidad en un estudio de intervención en que el niño era invitado a pensar sobre el significado del resto y sobre su relación con los otros términos. Cien niños (de 8 a 11 años), alumnos del $4^{\circ}$ año de primaria de escuelas públicas, después de una prueba previa, se asignaron en dos grupos homogéneos de acuerdo con las dificultades que presentaban con la división. El grupo experimental (GE) fue sometido a una intervención basada en los problemas de división inexacta que envolvía discusiones sobre las maneras apropiadas e inapropiadas de manejar el resto, y las relaciones de eso elemento con los otros términos de la división. La post-prueba comprobó que la intervención auxilió los alumnos do GE a superar sus dificultades iniciales con el resto. Al manejar el resto, el niño es llevado a establecer comparaciones entre el tamaño del restoyel número/tamaño de las partes, teniendo como principio la igualdad entre ellas, el principio fundamental de la división. Así, se comprende que el resto, cuando puesto en evidencia, se presenta como una herramienta didáctica capaz de ayudar al niño a desarrollar nociones más elaboradas sobre la división.

Palabras clave: Niños, división inexacta, resto, intervención.

Uma compreensão psicológica dos conceitos matemáticos, segundo Vergnaud (1990, 2003), requer considerar os invariantes lógicos e os esquemas de ação, as situações de uso e os suportes de representação. No caso do conceito de divisão (Nunes \& Bryant, 1997; Nunes, Campos, Magina, \& Bryant, 2001), os invariantes lógicos são: igualdade das partes em que o todo foi dividido; esgotar o todo até não ser possível outra rodada de distribuição; o todo inicial é constituído pelo número de partes multiplicado pelo tamanho das partes mais o resto; relação inversa entre o tamanho das partes e o número de partes; e o resto nunca pode ser maior nem igual ao número de partes ou tamanho das partes.

Pesquisas mostram que muitas das dificuldades em problemas de divisão decorrem da não compreensão das relações inversas entre os termos da divisão e de formas inadequadas de lidar com o resto (Correa, 1996; Correa, Nunes, \& Bryant, 1998; Kornilaki \& Nunes, 1997; Lautert \& Spinillo, 2004; Selva, 1998; Silver, 1988; Silver, Shapiro, \& Deutsch, 1993; Squire \& Bryant, 2002).

A presente investigação trata especificamente desta segunda dificuldade que tem marca- 
do muitas das resoluções incorretas adotadas em problemas de divisão inexata, como documentam os pesquisadores da área (Li \& Silver, 2000; Selva, 1998; Selva, Borba, Braga, \& Couto, 2004; Selva, Borba, \& Steedman, 2004; Silver, 1988; Silver et al., 1993).

Silver (1988) afirma que uma das dificuldades em lidar com o resto deve-se à diversidade de formas de representá-lo. Por exemplo, o resultado da operação $50 \div 4$ pode ser expresso como: $12.5,12$ 1/2 ou ainda 12 R2. Ainda, relata o autor, as formas de expressar o resto nem sempre são incorporadas à solução do problema.

Selva (1998) realizou uma ampla pesquisa acerca da divisão em crianças de 6 a 8 anos de idade. Dentre os objetivos propostos, a autora examinou se a forma de lidar com o resto variava em função dos tipos de problemas e/ou em função do material disponibilizado. As crianças foram divididas em três grupos: representação concreta (fichas), representação gráfica (papel e lápis) e sem material (cálculo mental). Foram apresentados problemas de divisão exata e inexata inseridos em um contexto de história. A autora constatou que a escolha das estratégias de resolução era influenciada pelo tipo de problema (se partição ou quotição), enquanto que o modo de lidar com o resto não. Ao analisar as respostas das crianças que mencionavam o resto, Selva identificou seis estratégias de lidar com esse elemento: (a) a criança solicitava uma quantidade maior de objetos para completar o resto, de modo a manter a igualdade entre as partes; (b) a criança colocava o resto em uma das partes, aceitando que esta parte ficasse com mais elementos que as demais; (c) a criança removia o resto; (d) a criança formava grupos iguais, independentemente do enunciado do problema; (e) a criança refazia o problema e; (f) a criança distribuía o resto entre todos os grupos. Os resultados demonstraram que as crianças menores tendem a aceitar a desigualdade das partes, enquanto que as crianças maiores, já instruídas sobre a divisão, optam ou pela remoção do resto ou por distribuí-lo entre todos os grupos.

Selva, Borba e Steedman (2004) investigaram se os tipos de problemas de divisão (partição e quotição) influenciavam no tratamento dado ao resto. Neste estudo, 32 crianças alunas do $3^{\circ} \mathrm{e}$ $4^{\circ}$ anos do ensino fundamental foram solicitadas a resolver problemas de divisão inexata, usando fichas. As principais estratégias adotadas em ambos os anos escolares foram: subdividir o resto em partes suficientes para uma nova redistribuição; acrescentar elementos ao quociente ou ignorar os elementos do resto. Os resultados indicaram que o tratamento dado ao resto é influenciado pelo tipo de problema: a estratégia de subdividir o resto foi típica dos problemas de partição, enquanto a estratégia de acrescentar elementos foi típica dos problemas de quotição. Não foram observadas diferenças na escolha das estratégias em função do tamanho do resto.

Um estudo com crianças alunas do ensino fundamental foi conduzido por Selva e Borba (2005) com o objetivo de explorar o tratamento dado ao resto e sua representação em decimais. Após um pré-teste que consistiu na resolução de seis problemas de divisão inexata (com lápis e papel), as 48 crianças investigadas foram distribuídas em três grupos que resolviam problemas de divisão inexata a partir do uso de diferentes suportes de representação: Grupo 1 - uso de papel e lápis seguido do uso de calculadora; Grupo 2 - uso de calculadora seguido do uso de papel e lápis; Grupo 3 - uso de fichas seguido do uso de papel e lápis. Posteriormente, as crianças foram submetidas a um pós-teste semelhante ao pré-teste. Observou-se que todos os grupos apresentaram um desempenho melhor no pós-teste do que no pré-teste; no entanto, em relação ao tratamento dado ao resto, não houve avanço, embora se tenha verificado que no pós-teste as crianças, sobretudo as mais velhas alunas do $6^{\circ}$ ano, passaram a mencioná-lo mais frequentemente. Segundo nossa análise, este resultado sugere que o suporte de representação utilizado durante a resolução dos problemas por si mesmo não teve qualquer efeito facilitador no modo da criança lidar com o resto. A pergunta que se faz, portanto, é: Que outras situações poderiam ser proporcionadas às crianças, de forma que elas pudessem lidar com o resto de maneira mais apropriada?

Spinillo, em estudos acerca do raciocínio combinatório em crianças (Spinillo \& Silva, 2010), acerca do conceito de medidas (Spinillo \& 
Batista, 2009) e a respeito do conceito de divisão (Lautert \& Spinillo, 2011; Lautert, Spinillo, \& Correa, 2012; Spinillo \& Lautert, 2006a, 2006b) considera que uma forma de desenvolver a compreensão acerca de diversos conceitos matemáticos é chamar a atenção da criança para os princípios invariantes que constituem o conceito em questão. Considerando que, como mencionado no início deste artigo, os invariantes lógicos da divisão envolvem as relações estabelecidas entre os seus termos, é possível que uma forma de desenvolver a compreensão acerca deste conceito seja chamar a atenção sobre o significado do resto (que é um de seus termos) na resolução de problemas de divisão inexata. Assim, a presente investigação examinou esta possibilidade em um estudo de intervenção em que, a partir da resolução de problemas de divisão inexata, a criança era encorajada a refletir sobre o significado do resto e sobre sua relação com os demais termos da divisão (divisor, dividendo e quociente). De maneira geral, o objetivo deste estudo foi desenvolver a compreensão da criança sobre a divisão, focalizando em especial a dificuldade em relação à forma de lidar com o resto, uma vez que a literatura aponta como sendo este um dos principais desafios observados na resolução de problemas de divisão. Como é usual em estudos de intervenção no campo da Psicologia do Desenvolvimento Cognitivo (Spinillo \& Lautert, 2008), esta investigação examinou a possibilidade de promover mudanças na compreensão de determinado fenômeno, no caso, o conceito de divisão.

\section{Método}

\section{Participantes}

Cem crianças de baixa renda, com idade entre 8 e 11 anos, alunas do $4^{\circ}$ ano do Ensino Fundamental de escolas públicas na cidade do Recife que apresentavam dificuldades com a divisão, conforme identificado em uma avaliação prévia aplicada a 206 crianças. Esta avaliação consistia na resolução de 12 problemas de divisão (partição e quotição), apresentados por escrito. Os participantes foram igualmente divididos em um Grupo Controle (GC) e um Grupo Experimental (GE), sendo pareados quanto à idade (média: 9 anos e 10 meses) e quanto ao nível de dificuldade que apresentavam quanto à divisão.

\section{Procedimento e Planejamento Experimental}

As crianças do Grupo Experimental (GE) individualmente participaram de uma intervenção conforme descrita adiante; enquanto as crianças do Grupo Controle (GC) continuaram apenas com o ensino usual da divisão em sala de aula. Os dois grupos realizaram um pré e um pós-teste, descritas a seguir.

O Pré-Teste e o Pós-Teste. O pré-teste e o pós-teste, individualmente aplicados, consistiam na resolução de seis problemas de divisão inexata (sendo três de partição e três de quotição), apresentado um por vez, com suas respectivas formas de resolução representadas pictograficamente. Era dada à criança a seguinte instrução: "Eu dei esse problema para um aluno de outra escola resolver e ele resolveu corretamente, desse jeito, como mostra essa cartela". Apresentava-se, então, o problema em uma cartela com a forma de resolução adotada (Figuras 1, 2 e 3).
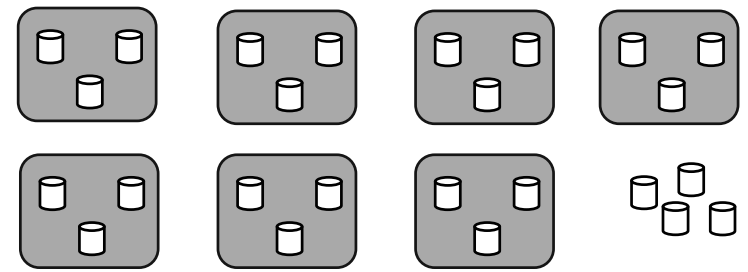

Figura 1. Marcos tem 25 copos e quer colocá-los em 7 bandejas. Ele quer que cada bandeja tenha a mesma quantidade de copos. Quantos copos ele irá colocar em cada bandeja? O que são esses copos ao lado? (Problema de divisão por partição).

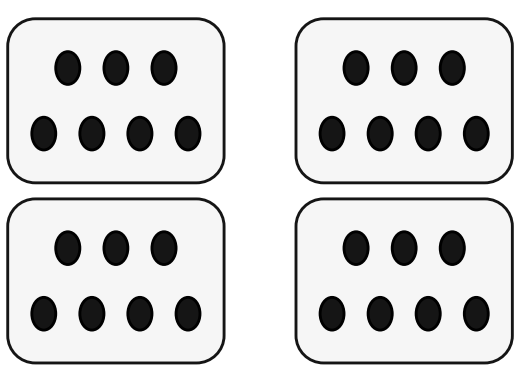

Figura 2. João tem 29 bolas de gude e quer colocá-las em 4 caixas. Ele quer que cada caixa tenha a mesma quantidade de bolas de gude. Quantas bolas de gude ele irá colocar em cada caixa? O que é essa bola de gude aqui ao lado? (Problema de divisão por partição). 

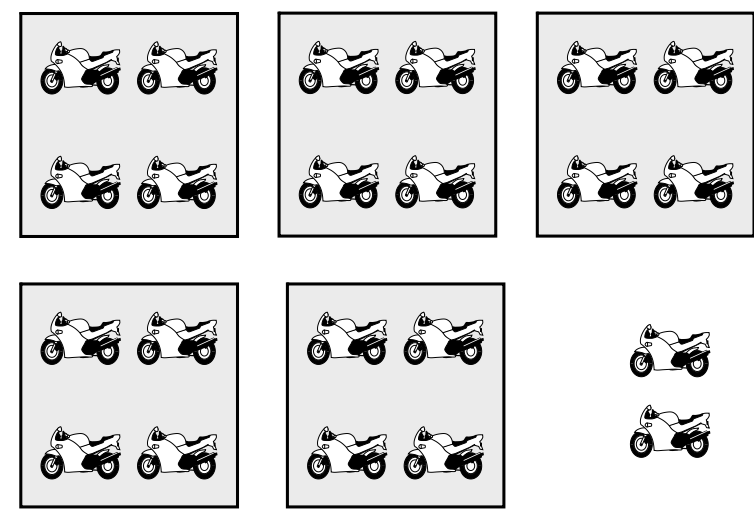

Figura 3. Luciano tem 22 motos de brinquedo. Ele quer guardar 4 motos em cada caixa. Quantas caixas ele irá precisar? O que são essas motos aqui ao lado? (Problema de divisão por quotição).

Os pares numéricos dos problemas no pré e no pós-testes foram $23: 5 ; 25: 7 ; 29: 4 ; 32: 7 ; 31: 5$ e $22: 4$. Os problemas no pós-teste eram análogos aqueles apresentados no pré-teste, diferindo apenas quanto aos referentes para as quantidades presentes nos enunciados dos problemas. As duas ocasiões de testagem foram gravadas e transcritas para posterior análise.

\section{A Intervenção}

A intervenção ocorreu em duas sessões aplicadas individualmente, com um período de dois a três dias entre elas. Em cada sessão foram apresentadas duas atividades em que, a partir de situações-problema, as relações entre os termos da divisão e o significado do resto eram colocadas em evidência. Os problemas eram apresentados por escrito, um por vez, sendo lidos pela examinadora juntamente com a criança. Para sua resolução eram fornecidos lápis e papel, objetos relativos aos referentes das quantidades presentes no enunciado dos problemas (copos e bandejas, estrelinhas e caixas, motos e caixas, bolas de gude e caixas, etc.).

Primeira Sessão. A Atividade 1 centrava-se na ideia de que o resto faz parte do processo de resolução de tal forma que ao se alterar o seu valor alteram-se os valores dos demais termos. $\mathrm{Na}$ Atividade 2 refletia-se sobre o resto a partir das relações entre o número de partes e o tamanho das partes quando o dividendo é mantido constante. As atividades são brevemente descritas a seguir.
1. Atividade 1: Alterando o valor do resto. Era apresentado um problema de base, do qual se derivavam outros problemas a partir de variações que eram feitas sobre o tamanho do resto. Exemplo 1: Ana comprou 22 botões e quer colocá-los em 4 caixas. Ela quer que cada caixa tenha a mesma quantidade de botões. Quantos botões ficarão em cada caixa? (problema de base: partição)

Variação 1: aumenta o resto, faz uma rodada de distribuição, obtendo resto igual a um. Examinadora: "E se a gente der mais três botões para Ana, como ficará a divisão dos botões nas caixas? [Acrescenta três botões ao resto]. Muda a resolução do problema? O que muda na resolução?"

Variação 2: aumenta o resto e, após nova rodada, obtém resto maior que um.

Examinadora: "E se a gente der mais cinco botões para Ana, como ficará a divisão dos botões nas caixas? [Acrescenta cinco botões ao resto]. Muda a resolução do problema? $\mathrm{O}$ que muda na resolução?"

Variação 3: aumenta o resto e, após nova rodada, obtém resto igual a zero.

Examinadora: "E se a gente der dois botões para Ana, como ficará a divisão dos botões nas caixas? [Acrescenta dois botões ao resto]. Muda a resolução do problema? O que muda na resolução?"

A examinadora explicitava que ao alterar o valor do resto alterava-se também o valor do dividendo e o valor do quociente. Ao final, mencionava os princípios invariantes da divisão, explicando que o resto nunca pode ser nem igual e nem maior que o número de partes, e nem igual e nem maior que o tamanho das partes precisando ser redistribuído igualmente entre as partes. Mencionava, ainda, que quando isso acontecia um novo resto seria produzido ou poderia ocorrer de o problema passar a ser um problema de divisão exata (resto igual a zero).

2. Atividade 2: Analisando procedimentos incorretos de resolução. Nesta atividade, a criança era solicitada a refletir sobre procedimentos de resolução que lhe eram apresentados a partir de materiais concretos 
manipulados pela examinadora. Era dito à criança que os procedimentos de resolução estavam incorretos e que haviam sido realizados por alunos (fictícios) de outra escola. Os procedimentos correspondiam a tipos de erros usualmente documentados na literatura, a saber: Erro 1: o resto era ignorado durante o processo de resolução; Erro 2: o resto era todo inserido em uma das partes; Erro 3: o resto era distribuído em algumas das partes, mas não em todas; Erro 4: o resto era maior que o número de partes. Note-se que esses tipos de erros violam os princípios invariantes da divisão anteriormente mencionados.

A instrução dada pode ser assim resumida: Eu dei um problema de divisão para um aluno de outra escola e ele resolveu deste jeito [examinadora mostra a partir de objetos, como o problema fora resolvido pelo aluno fictício]. Este jeito está incorreto. Qual foi o erro que o aluno fez?

A examinadora fornecia explicações e feedback após a resposta do participante.

Exemplo 2: Elena comprou 19 garrafas de refrigerante para a festa de aniversário de João. Ela quer servir 6 garrafas de refrigerante em cada bandeja. Quantas bandejas ela vai precisar? A criança da outra escola fez assim. O que foi que ela errou?

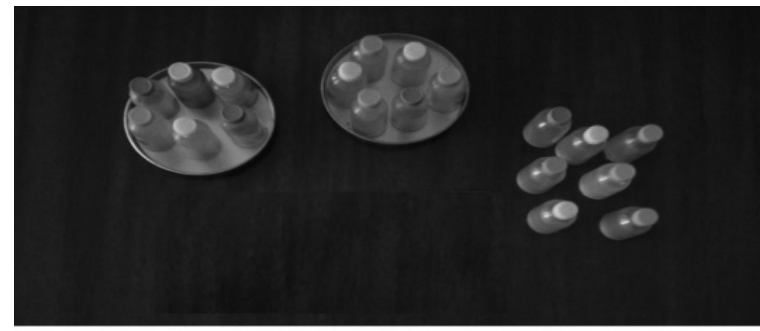

Figura 4. Erra porque o resto é maior do que o número de partes.

Segunda Sessão. Duas atividades foram apresentadas, as quais requeriam identificar e compreender os procedimentos corretos e incorretos de resolução em problemas de divisão exata e inexata que eram apresentados às crianças; e, no caso de procedimentos incorretos a criança era solicitada a propor formas mais adequadas de resolução do problema. Assim como na Atividade 2, os procedimentos incorretos apresentados violavam os princípios invariantes da divisão.

3. Atividade 3: Identificando procedimentos adequados de resolução. Era apresentado um problema e dois procedimentos de resolução (um correto e outro incorreto), solicitando-se que a criança identificasse qual aquele que julgava estar correto. A instrução pode ser assim resumida:

Eu dei um problema para duas crianças de outra escola resolver: Ana e Bruno. Essa cartela é como se fosse uma fotografia da forma como eles resolveram o problema. Eu vou mostrar o problema que eles resolveram e você irá dizer quem resolveu melhor, se foi Ana ou se foi Bruno.

Exemplo 3: Carlos comprou 34 foguetes e quer colocá-los em 6 caixas. Ele quer que cada caixa tenha a mesma quantidade de foguetes. Quantos foguetes ele irá colocar em cada caixa? Quem fez a melhor distribuição Ana ou Bruno?

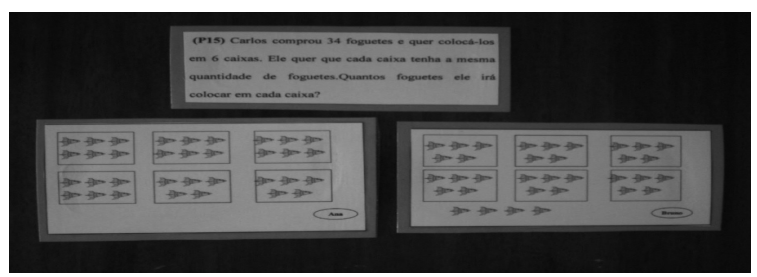

Figura 5. Ana (esquerda) erra porque seu procedimento viola a igualdade entre as partes.

Após a criança escolher e explicitar as razões de sua escolha, a examinadora fornecia feedback e explicações acerca das razões pelas quais um procedimento ser mais adequado que o outro. Especial ênfase foi dada à necessidade de manter a igualdade entre as partes e de redistribuir os elementos do resto de forma que este nunca resultasse em uma quantidade maior e nem igual ao número de partes ou ao tamanho das partes em que o todo havia sido dividido.

4. Atividade 4: Refletindo sobre procedimentos incorretos de resolução. Semelhante à Atividade 3, sendo que nesta atividade mostrava-se um procedimento incorreto e solicitava-se que a criança identificasse o tipo de erro e propusesse formas apropria- 
das de resolução do problema. Os tipos de erros eram os mesmos apresentados na atividade anterior. A instrução dada pode ser assim resumida:

Eu pedi para Luana, que é uma aluna de outra escola, resolver um problema e ela resolveu desse jeito, ela errou o problema. Eu queria que você me dissesse qual foi o erro que ela fez. Depois, quando você descobrir qual foi o erro, eu queria que você descobrisse como poderia fazer para resolver o problema de forma certa.

Exemplo 4: Débora comprou 29 bonecas para dar a suas 9 amigas. Ela quer que cada uma das amigas receba a mesma quantidade de bonecas. Quantas bonecas cada amiga vai receber?

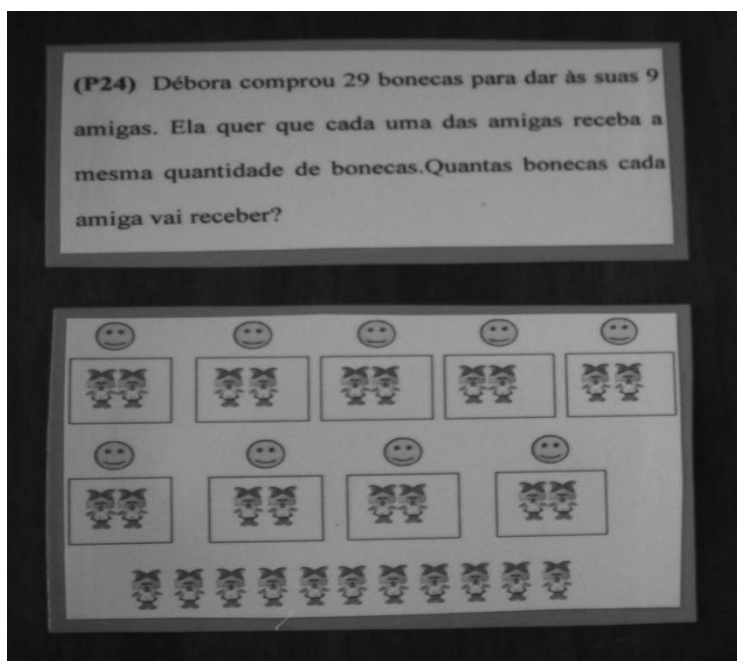

Figura 6. Luana erra porque o resto está maior do que o número de partes.

Ao final explicava-se a natureza do erro, mencionando-se os princípios invariantes da divisão que estavam sendo violados; bem como a examinadora fornecia feedback a respeito da solução proposta pela criança.

\section{Análise dos Dados e Resultados}

As respostas no pré e no pós-testes foram classificadas em três tipos, classificação esta que tomou por base os princípios invariantes dos conceitos matemáticos (Vergnaud, 1990, 1997, 2003) e os invariantes específicos ao conceito de divisão, conforme documentado na literatura (Nunes \& Bryant, 1997; Nunes et al., 2001). Os tipos de respostas identificados são descritos e exemplificados a seguir:

\section{Tipo 1}

Ausência de resposta ou respostas imprecisas que não permitem identificar qual o significado atribuído ao resto. Essas respostas foram dadas apesar da insistência da examinadora para obter informações mais claras por parte do entrevistado. Exemplo:

Exemplo 5: Marcos tem 25 copos e quer colocá-los em 7 bandejas. Ele quer que cada bandeja tenha a mesma quantidade de copos. (Figura 1).

E- Quantos copos ele irá colocar em cada bandeja?

C- Um.

E- No problema que ela [a criança fictícia] resolveu, o que são esses copos ao lado?

C- Correção.

E- Como assim?

C- Das bolas todas [interpreta o desenho dos copos como bolas].

\section{Tipo 2}

Resposta que viola o princípio da igualdade entre as partes. A criança procura formar uma nova parte para inserir o resto, ou insere todo o resto em uma parte já existente ou tenta distribuir os elementos do resto entre as partes já existentes. Seja de uma maneira ou de outra, ao adotar tal procedimento a criança gera partes de tamanhos distintos.

Exemplo 6: João tem 29 bolas de gude e quer colocá-las em 4 caixas. Ele quer que cada caixa tenha a mesma quantidade de bolas de gude. (Figura 2).

E- "Quantas bolas de gude ele irá colocar em cada caixa?"

C- "Sete em cada".

E- "No problema que foi resolvido, o que é essa bola de gude ao lado?"

C- "Essa aqui ele esqueceu de botar na caixa".

No Exemplo 6, criança insere a bola de gude em uma das caixas já existentes, ainda que esta 
decisão viole o princípio da igualdade das partes. Procedimentos como esse podem decorrer das situações cotidianas extraescolares que são resolvidas desta maneira.

\section{Tipo 3}

Resposta associada aos princípios invariantes da divisão.

Exemplo 7: Luciano tem 22 motos de brinquedo. Ele quer guardar 4 motos em cada caixa. (Figura 3).

E- Quantas caixas ele irá precisar?

C- Cinco.

E- No problema que ela [a criança fictícia], resolveu o que são essas motos ao lado?
C- $\mathrm{O}$ resto que não pode ficar dentro da caixa, senão vai ficar a quantidade diferente.

Dois juízes independentes analisaram as respostas obtidas, havendo $91,1 \%$ de concordância entre eles, sendo os julgamentos discordantes analisados por um terceiro juiz, também independente, cuja classificação foi considerada final.

Os dados no pré-teste foram submetidos a uma ANOVA, observando-se que o desempenho dos grupos não se diferencia tanto em relação aos problemas de divisão por partição $[F(1,98)$ $=1,524 ; p=0,220]$ como de quotição $[F(1,98)$ $=0,090 ; p=0,765]$, como mostra a Tabela 1 .

Tabela 1

Média de Acertos e Desvio Padrão (entre parênteses) em Função do Grupo e Tipo de Problema no Pré-Teste e no Pós-Teste

\begin{tabular}{|c|c|c|c|c|}
\hline & \multirow{2}{*}{ Grupos } & \multicolumn{2}{|c|}{ Tipos de problema } & \multirow[b]{2}{*}{ Total } \\
\hline & & Partição & Quotição & \\
\hline \multirow{2}{*}{ Pré-teste } & GC & $\begin{array}{c}1,66 \\
(1,189)\end{array}$ & $\begin{array}{c}1,64 \\
(0,942)\end{array}$ & $\begin{array}{c}1,65 \\
(1,066)\end{array}$ \\
\hline & GE & $\begin{array}{c}1,94 \\
(1,077)\end{array}$ & $\begin{array}{c}1,70 \\
(1,055)\end{array}$ & $\begin{array}{c}1,82 \\
(1,066)\end{array}$ \\
\hline \multirow{2}{*}{ Pós-teste } & $\mathrm{GC}$ & $\begin{array}{c}2,14 \\
(1,195)\end{array}$ & $\begin{array}{c}1,88 \\
(1,172)\end{array}$ & $\begin{array}{c}2,01 \\
(1,184)\end{array}$ \\
\hline & GE & $\begin{array}{c}2,82 \\
(0,438)\end{array}$ & $\begin{array}{c}2,78 \\
(0,465)\end{array}$ & $\begin{array}{c}2,80 \\
(0,452)\end{array}$ \\
\hline
\end{tabular}
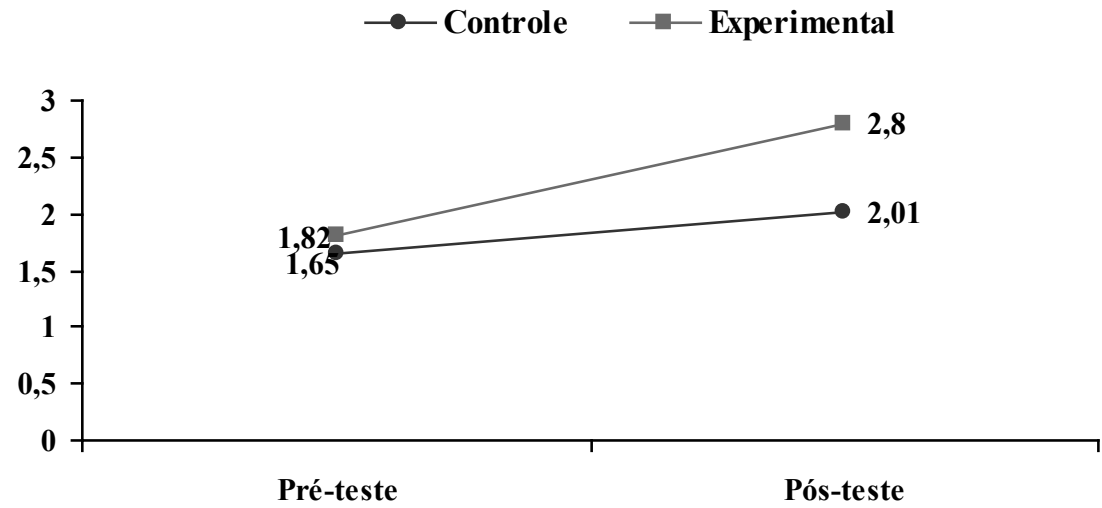

Figura 7. Média de acertos em cada grupo nas duas ocasiões de testagem. 
A interação ocasião de Testagem X Grupo foi significativa $[F(1,98)=18,649 ; p=0,001]$. De acordo com o Teste Tukey, isso ocorreu porque no pós-teste o número de acertos foi maior no GE do que no GC $(p<0,001)$ e que ambos os grupos tiveram um melhor desempenho no pós-teste do que no pré-teste $(p<0,001)$. No entanto, o aumento no número de acertos foi mais expressivo no GE do que no GC (Figura 7).

Comparações entre os grupos nas duas ocasiões de testagem foram exploradas a partir de uma ANOVA aplicada a cada tipo de resposta separadamente. Como mostra a Tabela 2, a única diferença encontrada foi em relação às respostas Tipo 3 no pós-teste que eram mais frequentes no GE do que no GC. Importante ressaltar que respostas Tipo 3 eram aquelas que expressavam uma compreensão acerca do significado do resto, respeitando os princípios invariantes da divisão.

A interação ocasião de Testagem X Grupo, em relação às respostas Tipo 3 , foi significativa $[F(1,98)=38,642 ; p=0,001]$. Como se observa na Figura 8, confirmado pelo Teste de Tukey, há diferenças significativas entre os grupos no pós-teste $(p<0,001)$, visto que as crianças do GE forneciam mais respostas Tipo 3 do que as do GC (médias: 2,84 e 1,68, respectivamente).

Tabela 2

Média de Respostas em Cada Grupo e nas Duas Ocasiões de Testagem

\begin{tabular}{ccccc}
\hline & \multicolumn{3}{c}{ Pré-teste } & \multicolumn{2}{c}{ Pós-teste } \\
\cline { 2 - 5 } Respostas & GC & GE & GC & GE \\
\hline $\begin{array}{c}\text { Tipo 1 } \\
\text { (ausente, imprecisa) }\end{array}$ & 1,10 & 0,95 & 0,91 & 0,11 \\
$\begin{array}{c}\text { Tipo 2 } \\
\text { (viola a igualdade) }\end{array}$ & 0,40 & 0,58 & 0,41 & 0,05 \\
Tipo 3 \\
(considera os invariantes)
\end{tabular}

$\multimap$ Controle $\longrightarrow-$ Experimental

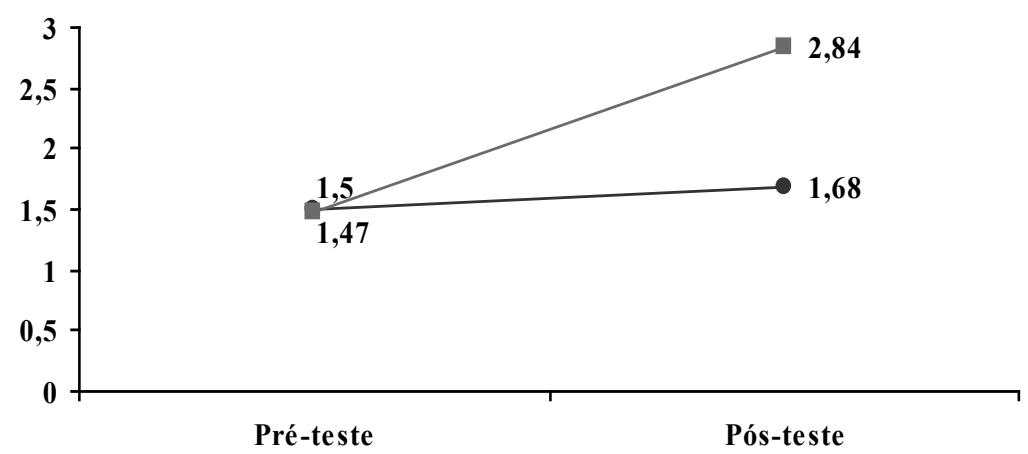

Figura 8. Média de respostas Tipo 3 em cada grupo nas duas ocasiões de testagem.

O Teste de Tukey detectou diferenças significativas entre o pré e o pós-teste no GE ( $p<$ $0,001)$, pois a média de respostas Tipo 3 no pós-teste era mais alta do que no pré-teste (Figura
8). No entanto, não foram observadas diferenças significativas entre as duas ocasiões de testagem no GC, sendo a média de respostas Tipo 3 igualmente baixa nas duas ocasiões. Este dado indica 
que apesar do GC haver também melhorado seu desempenho do pré para o pós-teste (Figura 7), este desempenho não estava associado a uma compreensão dos invariantes, compreensão esta presente nas respostas Tipo 3 que foram pouco frequentes neste grupo.
Os dados mostram que a intervenção teve papel facilitador na compreensão da divisão, tanto em relação ao desempenho como em relação aos tipos de respostas. No entanto, é importante examinar a natureza da progressão quando se compara o pré-teste e o pós-teste nos dois grupos em relação aos tipos de respostas (Tabela 3).

Tabela 3

Porcentagem de Cada Tipo de Progressão Identificado do Pré-Teste para o Pós-Teste em Cada Grupo

\begin{tabular}{ccc}
\hline Progressão do pré para o pós-teste & $\begin{array}{c}\text { Grupo Controle } \\
(n=41)\end{array}$ & $\begin{array}{c}\text { Grupo Experimental } \\
(n=138)\end{array}$ \\
\hline Tipo $1 \rightarrow$ Tipo 2 & 35 & 63 \\
Tipo $1 \rightarrow$ Tipo 3 & 51 & 36,3 \\
Tipo $2 \rightarrow$ Tipo 3 & 14 & 36 \\
\hline
\end{tabular}

Nota. Tipo 1 - resposta ausente ou imprecisa; Tipo 2 - viola a igualdade; Tipo 3 - considera os invariantes adequadamente.

Inicialmente é importante comentar que $77,1 \%$ das progressões identificadas ocorreram no GE e apenas $22,9 \%$ no GC. Observa-se, ainda, que avanços mais substanciais, como de respostas Tipo 1 para Tipo 3, foram mais frequentes no GE (63\%) do que no GC (51\%), assim como as respostas do Tipo 2 para o Tipo 3 (GE: $36,3 \%$; GC: $14 \%)$. Na realidade, a quase totalidade das progressões no GE levavam as crianças a apresentarem respostas Tipo 3 (99,3\%). Esses resultados, ainda que tratados em termos de tendências, visto que nenhum tratamento estatístico foi aplicado, indicam que as crianças que participaram da intervenção passaram a adotar respostas que representavam avanços bem mais expressivos do que as crianças do GC.

\section{Conclusões e Discussão}

$\mathrm{Na}$ presente investigação, as dificuldades das crianças em relação à forma de lidar com o resto foram tratadas em dois planos. Em um primeiro plano, procedeu-se a um levantamento das formas inapropriadas que as crianças adotam ao lidar com o resto em problemas de divisão inexata, como documentado na literatura. Ao compreender a natureza dos erros das crianças, foi possível compreender suas formas de racio- cinar e as dificuldades que enfrentam ao resolver problemas de divisão. Em um segundo plano, os erros das crianças passaram a fazer parte da intervenção nesta pesquisa, em especial, as formas inapropriadas de lidar com o resto, como fica evidente nas atividades proporcionadas ao grupo experimental. É possível dizer que durante a intervenção foi dado um tratamento didático ao erro, cuja importância é ressaltada por Cury (2007), por Pinto (2000), e por Spinillo, Pacheco, Ferreira e Cavalcanti (2014), que afirmam que a análise do erro pode ser uma estratégia didática a ser adotada em sala de aula. Este aspecto foi considerado nas situações em que as crianças eram solicitadas a pensar acerca de procedimentos incorretos de resolução. Esta estratégia pode tornar-se um recurso didático poderoso; uma ferramenta que, inserida em um ambiente de discussão e reflexão, pode possibilitar a compreensão de conceitos matemáticos diversos.

Esse tratamento didático foi associado a um referencial teórico que pressupõe que os conceitos matemáticos envolvem princípios invariantes que o definem (Vergnaud, 1997). No caso do conceito de divisão, esses princípios envolvem considerar o resto e sua relação com os demais termos da divisão, como comentado no início deste artigo. No entanto, muitas vezes 
esses princípios são violados, levando a criança ao erro. Em decorrência disso, a intervenção forneceu um tratamento didático ao erro associado à ideia de que os princípios invariantes de um dado conceito podem ser explicitados em um contexto de resolução de problemas e, assim, refletidos por aquele que soluciona o problema. Tal intervenção auxiliou as crianças a adotar formas apropriadas de lidar com o resto e, consequentemente, a ter uma maior compreensão acerca da divisão.

De fato, os resultados obtidos neste estudo mostraram que após a intervenção as crianças do grupo experimental alcançaram níveis de compreensão mais sofisticados sobre os invariantes operatórios da divisão do que as crianças do grupo controle, apesar de ambos os grupos terem apresentado o mesmo nível de dificuldade na ocasião do pré-teste. Esta compreensão não ficou circunscrita apenas ao desempenho (número de acertos), sendo também observada em relação aos tipos de respostas fornecidas aos problemas. Enquanto as crianças do grupo controle continuavam a oferecer respostas imprecisas a respeito do resto ou a associar o resto com soluções que violavam alguns dos princípios invariantes da divisão, as crianças do grupo experimental após a intervenção estavam conscientes da necessidade de garantir a igualdade entre as partes e conscientes de que o resto não poderia ser maior do que o número de partes ou maior do que o tamanho das partes. De acordo com nossa análise, para compreender o significado do resto é importante tomar consciência da existência da igualdade entre as partes e da necessidade de não violar este princípio.

Outra ideia que merece ser discutida a partir dos dados deste estudo refere-se ao fato de que ao manipular o resto, a criança é levada a estabelecer comparações entre o tamanho do resto e o número/tamanho das partes, tendo como âncora o princípio da igualdade entre as partes. Neste sentido, é possível dizer que o resto pode ser um termo da divisão capaz de auxiliar a criança a desenvolver noções mais elaboradas sobre este conceito. Assim, problemas de divisão inexata deveriam ser introduzidos desde muito cedo no currículo do ensino fundamental, inserido em si- tuações em que o resto é colocado em evidência durante o processo de resolução.

Um último aspecto a comentar, porém não de menor relevância, refere-se à interação adulto-criança durante a intervenção. A interação foi marcada por discussões e reflexões de natureza metacognitiva que levavam a criança a refletir acerca dos seus processos de resolução, de sua forma de raciocinar frente a uma dada situação-problema. A metacognição, enquanto mecanismo cognitivo pode facilitar a aprendizagem, como ressaltam Lautert e Spinillo (2011) e Ribeiro (2003). Neste sentido, a sala de aula poderia tornar-se um ambiente marcado por atividades de natureza metacognitiva em que o aluno é solicitado a tomar seu pensamento e suas formas de raciocinar como objeto de reflexão e análise.

\section{Referências}

Correa, J. (1996). A compreensão inicial do conceito de divisão partitiva em tarefas não-computacionais. In M. H. Novaes \& M. R. F. Brito (Eds.), Psicologia na Educação: Articulação entre pesquisa, formação e prática pedagógica. Coletâneas da ANPEPP: Vol. 1 (pp. 151-165). Rio de Janeiro, RJ: Xenon.

Correa, J., Nunes, T., \& Bryant, P. (1998). Young children's understanding of division: The relationship between division terms in a noncomputational task. Journal of Educational Psychology, 90(2), 321-329. doi:10.1037/00220663.90.2.321

Cury, H. N. (2007). Análise de erros o que podemos aprender com as respostas dos alunos. Belo Horizonte, MG: Autêntica.

Kornilaki, E., \& Nunes, T. (1997). What do young children understand about division? In_Proceedings of the $21^{\text {th }}$ Annual International Conference of Psychology of Mathematics Education (Vol. 1, p. 242). Lahti, Finland: University of Helsinki.

Lautert, S. L., \& Spinillo, A. G. (2004). Inverse relations between division terms: A difficulty children are able to overcome In Proceedings of the 28 $8^{\text {th }}$ Annual International Conference of Psychology of Mathematics Education (Vol. 1, p. 397). Bergen, Norway: Bergen University College. 
Lautert, S. L., \& Spinillo, A. G. (2011). Estudo de intervenção sobre a divisão: Ilustrando as relações entre metacognição e aprendizagem. $E d u$ car em Revista, 1, 93-107. doi:10.1590/S010440602011000400007

Lautert, S. L., Spinillo, A. G., \& Correa, J. (2012). Children's difficulties with division: An intervention study. Journal of Medicine and Medical Sciences, 1, 447-456.

Li, Y., \& Silver, E. A. (2000). Can younger students succeed where older students fail? An examination of third graders'solutions of a divisionwith-remainder (DWR) problem. Journal of Mathematical Behavior, 19, 233-246. doi:10.1016/ S0732-3123(00)00046-8

Nunes, T., \& Bryant, P. (1997). Crianças fazendo Matemática. Porto Alegre, RS: Artes Médicas.

Nunes, T., Campos, T. M. M., Magina, S., \& Bryant, P. (2001). Introdução à Educação Matemática: Os números e as operações numéricas. São Paulo, SP: Programa de Bolsas para Alunos do Ensino Médio em Escolas Públicas.

Pinto, N. B. (2000). O erro como estratégia didática. Estudo do erro no ensino da Matemática Elementar. São Paulo, SP: Papirus.

Ribeiro, C. (2003). Metacognição: Um apoio ao processo de aprendizagem. Psicologia Reflexão e Crítica, 16(1), 109-116.

Selva, A. C. V. (1998). Discutindo o uso de materiais concretos na resolução de problemas e divisão. In A. Schliemann \& D. Carraher (Eds.), A compreensão de conceitos aritméticos. Ensino e pesquisa (pp. 95-119). Campinas, SP: Papirus

Selva, A. C. V., \& Borba, R. E. S. (2005). O uso de diferentes representações na resolução de problemas de divisão inexata: Analisando a contribuição da calculadora. Boletim do Grupo de Estudos e Pesquisas em Educação Matemática, 47, 51-72.

Selva, A. C. V., Borba, R. E. S., Braga, J. T., \& Couto, T. S. (2004). Como calculadora pode ser usada em sala de aula: Um estudo exploratório. In Anais VIII Encontro Nacional de Educação Matemática [CD-ROM]. Recife, PE: Sociedade Brasileira de Educação Matemática.

Selva, A. C. V., Borba, R. E. S., \& Steedman, L. H. S. (2004). Explorando a resolução de problemas de divisão com resto por crianças de $2^{\mathrm{a}}$ e $3^{\mathrm{a}}$ séries. In Anais VIII Encontro Nacional de Educação
Matemática [CD-ROM]. Recife, PE: Sociedade Brasileira de Educação Matemática.

Silver, E. A. (1988). Solving story problems involving division with remaiders: The importance of semantic processing and referential mapping. In Proceedings of the $10^{\text {th }}$ Annual Meeting of the North American Chaper of the International Group for the Psychology of Mathematics Education (pp. 127-133). DeKalb, IL: North Illinois University.

Silver, E., Shapiro, L. J., \& Deutsch, A. (1993). Sense making and the solution of division problems involving remainders: An examination of middle school students' solution processes and their interpretations of solutions. Journal for Research in Mathematics Education, 24(2), 117-135. doi: $10.2307 / 749216$

Spinillo, A. G., \& Batista, R. M. F. (2009). A sense of measurement: What do children know about the invariant principles of different types of mesurement. In Proceedings of the $33^{\text {th }}$ Annual International Conference of Psychology of Mathematics Education (Vol. 5, pp. 161-168). Thessaloniki, Greece: International Group for the Psychology of Mathematics Education.

Spinillo, A. G., \& Lautert, S. L. (2006a). Exploring the role played by the remainder in the solution of division problems. In Proceedings of the $30^{\text {th }}$ Annual International Conference of Psychology of Mathematics Education (Vol. 5, pp. 153-162). Prague, Czech Republic: International Group for the Psychology of Mathematics Education.

Spinillo, A. G., \& Lautert, S. L. (2006b). O diálogo entre a Psicologia do Desenvolvimento Cognitivo e a Educação Matemática. In L. Meira \& A. G. Spinillo (Eds.), Psicologia Cognitiva: Cultura, desenvolvimento e aprendizagem (pp. 46-80). Recife, PE: Editora da Universidade Federal de Pernambuco.

Spinillo, A. G., \& Lautert, S. L. (2008). Pesquisa de intervenção em Psicologia do Desenvolvimento Cognitivo: Reflexões e resultados. In L. R. de Castro \& V. L. Besset. (Eds.), Pesquisa de intervenção na infância e adolescência (pp. 294321). Rio de Janeiro, RJ: Nau.

Spinillo, A. G., Pacheco, A., Ferreira, J., \& Cavalcanti, L. (2014). O erro no processo de ensino-aprendizagem da matemática: Errar é preciso? Boletim GEPEM (Online), v. 64, p. $1-12,2014$ 
Spinillo, A., \& Silva, J. F. G. (2010). Making explicit the principles governing combinatorial reasoning: Does it help children to solve cartesian product problems? In Proceedings of the $34^{\text {th }}$ Conference of the International Group for the Psychology of Mathematics Education (Vol. 4, pp. 216-224). Belo Horizonte, MG: International Group for the Psychology of Mathematics Education.

Squire, S., \& Bryant, P. (2002). The influence of sharing of children's initial concept of division. Journal for Experimental Child Psychology, 81, 1-43. doi:10.1006/jecp.2001.2640

Vergnaud, G. (1990). La théorie des champs conceptuels. Recherches en Didactique des Mathématiques, 10(13), 133-170.
Vergnaud, G. (1997). The nature of mathematical concepts. In T. Nunes \& P. Bryant (Eds.), Learning and teaching mathematics: An international perspective (pp. 5-28). Hove, UK: Psychology Press.

Vergnaud, G. (2003). A gênese dos campos conceituais In E. Grossi (Ed.), Por que ainda há quem não aprende? A teoria (pp. 21-60). Rio de Janeiro, RJ: Vozes. 